INNOVATIONS IN PRIMARY CARE

\title{
Moving From In-Person to Telehealth Group Visits for a Mindful-Eating Healthy Nutrition Program
}

\author{
Jennifer K. Carroll, MD, MPH \\ Leonard Finn, MD \\ Kirsten Scharer, RN \\ Lauren Kiel \\ Asbley Kiel \\ Elisabeth Callen, $\mathrm{PbD}$, GStat
}

Erin Callister

Ina Campbell

Emma Anderson, MA

Carrie Landin, PsyD
Lauren Grossman, MD, SM

Anne Nederveld, MD, MPH

Ann Fam Med 2021;19:466. https://doi.org/10.1370/afm.2685.

\section{THE INNOVATION}

The COVID-19 pandemic necessitated an unanticipated innovation: the transition from in-person to telehealth group visits in a mindful-eating healthy nutrition program.

\section{WHO AND WHERE}

We provide our group visit program at 2 locations: a family physician-owned practice (Needham Wellesley Family Medicine PC, Wellesley, Masschusetts) and an integrative medicine practice within an academic medical center health system (UC Health Integrative Medicine Center, Denver, Colorado).

\section{HOW}

The curriculum consists of 10 weekly 2 -hour sessions, followed by 3 monthly 2 -hour refresher sessions. Interested eligible individuals are recruited through referrals from primary care physicians, clinical colleagues, or self-referral. Staff screen and refer patients to verify eligibility and ensure understanding of the program. Weekly visits are facilitated by a physician and an experienced mindfulness teacher using a manualized mindfuleating healthy nutrition curriculum. During each session, participants discuss their goals, struggles, insights, and progress.

In May 2020, COVID-19 caused the Wellesley site to successfully ransition from in-person to telehealth visits via use of its HIPAA-compliant, institutionally approved telehealth platform (Zoom). Before each virtual visit, links to the telehealth session,

Conflicts of interest: authors report none.

\section{Corresponding author}

Jennifer K. Carroll

University of Colorado Department of Family Medicine

12631 E. 17th Ave, Mailstop F496

Aurora, CO 80045

jennifer.2.carroll@cuanschutz.edu pre-visit questionnaires, and teaching materials are e-mailed to patients. Based on the initial experience in Wellesley, the Denver site implemented telehealth group visits in early 2021.

\section{LEARNING}

In contrast to our independent site, start-up for our site within the large health system was labor- and time-consuming, with administrative, logistical, technological, and regulatory issues.

\section{Challenges}

Clinicians and patients had to become familiar with the telehealth software, have access to a device with a camera and microphone, and have an adequate Internet connection. Virtual meeting environments may be less quiet than office space. Another challenge was learning to send questionnaires and education materials to patients, who must download and return them quickly. For patients unfamiliar with technology, we used postal mail, resulting in delays. An ongoing challenge is remotely measuring patients' weight, blood pressure, and waist circumference. Administrative challenges included scheduling, training staff, and navigating and procuring administrative, billing, documentation, and institutional review board (IRB) approvals.

\section{Benefits}

Overall, the transition to telehealth has been easier than anticipated. Telehealth benefits include no commute time, parking issues, stairs, or other accommodations; a more streamlined check-in and ability to send materials in advance; and no need for meeting space. It was easier to pair patients to share experiences in virtual breakout rooms than in a meeting room. Patient participation and interactivity were more robust than expected. Attendance and retention appreciably improved.

\section{Potential Losses}

Converting from in-person to telehealth changes some teaching materials. We also lost the ability to serve healthy snacks and chat before, during, and after the visit. Group dynamics are more difficult to appreciate. Participants with vision impairments could not attend sessions. Further, without full body language visible, telehealth visits require adaptation of communication skills such as adequate pauses to ensure a person has finished speaking.

Supplemental material (affiliations, key words) at https://www. AnnFamMed.org/lookup/suppl/doi:10.1370/afm.2685/-IDC1.

Submitted November 4, 2020; submitted, revised, December 18, 2020; accepted January 4, 2021. 